\title{
Herramientas digitales aplicadas en el escenario de la inclusión educativa en la época de la COVID-19
}

\section{Digital tools applied in the scenario of educational inclusion in the era of COVID-19}

\author{
Estela Isequilla Alarcón \\ Universidad de Málaga (España) \\ eisequilla@uma.es \\ María Martín-Delgado \\ Universidad de Málaga (España) \\ mariammdd@uma.es
}

Recibido:01/09/2021

Aceptado: $22 / 11 / 2021$

Publicado: 01/12/2021

\section{RESUMEN}

El presente estudio tiene por objeto conocer cuáles son las aplicaciones más utilizadas en el ámbito educativo desde que se declaró el estado de alarma, en marzo de 2020. Se aplicó un cuestionario en el cual participaron 65 docentes y 127 estudiantes de la provincia de Málaga. Los datos de éste reflejan que la muestra considerada se adecúa a los parámetros de muestra para proseguir con la investigación. Además, se utilizó el alfa de Cronbach ( $\alpha=.740$ y $\alpha=.658$, respectivamente) para determinar su validez y fiabilidad. Los resultados obtenidos muestran cómo el alumnado está más familiarizado con las aplicaciones de Google, frente al profesorado que opta por emplear otros softwares, como son las plataformas online. Concretamente, un mayor número de alumnado utilizaba la herramienta Google Meet para recibir sus clases no presenciales. Sin embargo, un elevado porcentaje ha preferido dar sus clases a través de Moodle Centros. Asimismo, la pandemia ha originado conocer otros recursos tecnológicos y aprender a cómo utilizar estos, como es el caso de Google Meet, para los estudiantes y Microsoft Teams, para los docentes. Todo ello, ha supuesto un avance para el sector educativo, proliferando todo el entramado tecnológico y de recursos online, intentando hacer llegar la educación a gran parte de la población para combatir la actual brecha digital existente en muchos hogares.

\section{PALABRAS CLAVE}

Estudiante, Educación a distancia; Software didáctico; Aplicación de telecomunicaciones; TIC.

\section{ABSTRACT}

The present study aims to know which are the most used applications in the educational field since the state of alarm was declared in March 2020. A questionnaire was applied in which 65 were teachers and 127 were students from the province of Málaga. The data from this reflect that the sample considered is adapted to the sampling parameters to continue with the investigation. In addition, Cronbach's alpha $(\alpha=$ .740 and $\alpha=.658$, respectively) was used to determine its validity and reliability. The results obtained show how students are more familiar with Google applications, compared to teachers who choose to use other software, such as online platforms. More specifically, a greater number of students used the Google Meet tool to receive their non-face-to-face classes. However, a high percentage have preferred to teach their classes through Moodle Centers. Likewise, the pandemic has led to knowing other technological resources and learning how to use them, such as Google Meet, for students and Microsoft Teams, for teachers. All this has meant progress for the education sector, proliferación the entire technological network and online 
resources, trying to bring education to a large part of the population to combat the current digital gap that exists in many homes.

\section{KEYWORDS}

Student; Long distance education; Educational software; Telecommunications application; ICT.

\section{CITA RECOMENDADA}

Isequilla, E. \& Martín-Delgado, M. (2021). Herramientas digitales aplicadas en el escenario de la inclusión educativa en la época de la COVID-19. RIITE Revista Interuniversitaria de Investigación en Tecnología Educativa, 11, 40-52. https://doi.org/10.6018/riite.490731

\section{Principales aportaciones del artículo y futuras líneas de investigación:}

- Intenta concienciar a la comunidad educativa y universitaria sobre la importancia de saber manejar y gestionar adecuadamente las aplicaciones digitales para impartir una docencia de calidad.

- Se observa un gran auge de las TIC en época de la Covid-19, en la cual los agentes educativos y el alumnado se vieron en la obligación de aprender a utilizar nuevas plataformas digitales.

- El profesorado se inclinó por determinadas TIC para impartir su materia didáctica, así como el alumnado tiene tendencia por otras entre las que destacan las redes sociales.

\section{INTRODUCCIÓN}

Hussin \& Siddappa (2020) indican que el pasado 14 de marzo de 2020, se decretó el estado de alarma en España, suponiendo la suspensión lectiva presencial de los centros educativos y de las universidades, debido a la Covid-19, ya que los sanitarios no sabían cómo hacer frente a este virus, el cual ha provocado innumerables muertes. La Covid-19 es una enfermedad que afecta al sistema respiratorio, está considerada como una amenaza para la salud pública. Se produjo a finales de diciembre de 2019, cuando algunos pacientes acudieron al hospital de Wuhan, provincia de Hubei (China) con un diagnóstico inicial de neumonía de etiología desconocida. Tuvieron que hospitalizar a cinco personas con problemas respiratorios agudos, uno de ellos perdió la vida. La autoridad sanitaria se percató de que era un virus mortal con el cual se contagiaban muchas personas. De ahí la razón de que se cerrara toda actividad no considerada esencial (García et al., 2020).

Así que el sector educativo se encontró ante uno de los mayores desafíos: hacer llegar la educación mediante la modalidad online a todos los niños, adolescentes y jóvenes que estaban formándose, hasta ese momento, presencialmente. Por consiguiente, el Gobierno hizo el siguiente comunicado: "las actividades educativas a través de las modalidades a distancia y "online", siempre que resulte posible" (Real Decreto 463/2020, de 14 de marzo porque se declara el estado de alarma para la gestión de la situación de crisis sanitaria ocasionada por la Covid19).

No todas las personas han tenido los medios digitales para seguir las clases online en época de la Covid-19, provocando una gran brecha digital en la educación. Por ende, muchos de ellos han tenido desinformación. Las TIC han tenido un papel fundamental para que las personas se socialicen entre ellas. La pandemia ha modificado la forma de vivir del ciudadano, ya que el trabajo se convirtió en teletrabajo, la educación se transformó en online y la comunicación con los amigos, familiares y profesores tuvo que ser a distancia (Kuric et al., 2020). Por lo tanto; se generó una dependencia tecnológica, originando nuevos grupos vulnerables por la desigualdad digital, siendo un elemento elemental de la jerarquización social (Robinson et al., 2020). 
De manera colateral, el profesorado ha tenido que reinventarse para dar sus clases, cambiando la forma pedagógica en la que solían hacerlo, aunque algunos de ellos discreparan de esta manera de concebir la enseñanza online, como otra forma diferente de enseñar. Muchos de ellos tuvieron que hacer un esfuerzo considerable, pues la gran mayoría no tiene una adecuada formación en el uso de las TIC. Se tiene que apreciar esta forma de enseñar como una oportunidad de aprendizaje, dado que tanto estudiantes como docentes tuvieron que aprender a controlar su estado emocional y trabajar los distintos aspectos de las asignaturas con las TIC, produciéndose un aprendizaje significativo (Cifuentes-Faura, 2020; Gregorio, 2020).

Es fundamental que el profesorado seleccione minuciosamente las aplicaciones digitales con la intención de mejorar la interacción con el alumnado. Las TIC han conseguido que se puedan impartir los contenidos programados en el currículum de la asignatura. Se debe resaltar el esfuerzo que tuvo que realizar el profesorado para innovar un contexto digital para que el alumnado adquiriera el aprendizaje, fomentando sus competencias digitales. Llegado este punto, el profesorado debía hacer un análisis sobre las aplicaciones digitales más adecuadas para impartir las asignaturas. No se debe olvidar que las TIC han representado un apoyo esencial para el proceso de enseñanza-aprendizaje en la docencia (Ramiro et al., 2020).

En consonancia con lo anterior, para Calero (2019) considera que no solo es suficiente con tener digitalizadas las aulas, en los centros educativos, si ésta no va acompañada de una fuerte innovación pedagógica que asegure el aprendizaje de todo el alumnado y, en buena medida, esto se debe a los estilos en que mejor aprende cada sujeto. Sin embargo, el modelo pedagógico no debería ser distinto al que se desarrolla de forma presencial, ya que las Tecnologías de la Información y Comunicación (TIC) se utilizan como otro medio de conectar con el alumnado para hacer posible la enseñanza (Álvarez-Sigüenza, 2019, citado por Rodicio-García et al, 2020).

En línea con lo anterior, el docente ha tenido que afrontar una situación muy compleja, por la falta de recursos no concebidos a tiempo para frenar la brecha digital, que no se ha sabido prever en muchos hogares (Anguita et al., 2020). Por lo tanto, la educación sigue perfilándose de forma desequilibrada, no ajustándose al derecho que recoge el artículo 27 , sección 1a de los Derechos fundamentales y de las Libertades Públicas, capítulo 2. ํ. Derechos y Libertades y título $1^{\circ}$, de los Derechos y Deberes fundamentales de la Constitución española de 1978, en materia de educación. Éste se considera como uno de los fundamentos básicos de nuestro ordenamiento jurídico estatal e internacional, así como de los Derechos Humanos. En cuanto a la Ley Orgánica $3 / 2020$, de 29 de diciembre, por la que se modifica la Ley Orgánica 2/2006, de 3 de mayo, de Educación (LOMLOE) basada en principios como el respeto de los derechos de la infancia, la educación inclusiva, la equidad, la igualdad de género y la orientación educativa y profesional.

En suma, el derecho a la educación no solo es un concepto que se centra en los aprendizajes que puede recibir una persona. Éste supone un derecho holístico de la sociedad, puesto que con él se pueden valorar cómo esos aprendizajes contribuyen al desarrollo social del individuo y cómo se desenvuelve ante hechos históricos y culturales que están presentes también en la evolución del sujeto, de manera más particular (Ramón, 2020).

En este sentido, datos del Instituto Nacional Español (INE, 2020) muestra cómo el 4,6\% de los hogares de la población española no tienen conexión a Internet. Quizá, uno de los motivos principales sea la falta de recursos existentes en muchos hogares para cubrir sus necesidades más básicas, como la alimentación o el atuendo. Para una época que ha sido digitalizada en la mayoría de sus esferas sociales, estos datos deberían alarmar a los lectores y preocupar a aquellos agentes que invierten su ocupación en concebir una educación para todos.

Además de la latente brecha digital a nivel socio-cultural, de aptitudes tecnológicas y de infraestructura, según Alonso (2019) surgían otros problemas colaterales a los mismos, como la falta de enseñanza de sus progenitores que, aunque en algunos casos acompañaban a sus hijos en las clases online, no disponían del mejor entorno para su proceso de enseñanza-aprendizaje y en otros muchos casos, carecían de tiempo, herramientas y estrategias para lidiar con la estabilidad emocional que rápidamente podía mermarse a causa de la situación por la Covid-19 y que, por tanto, tampoco facilitaba los aprendizajes del alumnado (Martín, 2020). 


\subsection{La educación online: pasado, presente y futuro}

Tibi \& Tibi (2015) afirman como la enseñanza online ha sido posible en nuestros días, gracias a la llegada de Internet, lo que originó ya en su época un cambio para el ámbito educativo. Con el paso del tiempo, las cosas acaban siendo obsoletas y en pleno siglo XXI se ha podido desarrollar múltiples actividades que se solían practicar en el colegio, como, por ejemplo: realizar un taller de teatro con universitarios con discapacidad intelectual o una graduación online, como sello innovador de superar las limitaciones que de un primer momento se pensaba que podía haber ocasionado la Covid-19.

En línea con lo anterior, han sido muchas las empresas e instituciones que han proliferado en este tiempo; bien encontrando un nuevo nicho de mercado o, por el contrario, optimizando sus recursos para hacer crecer su servicio y aumentando así el coste-beneficio. Frecuentemente, se ha podido encontrar numerosos cursos que realizar, webinars o encuentros gratuitos a los que asistir, material descargable con el que se podía complementar la formación o un creciente número elevado de espectadores que consumían recursos visuales con los que ser autodidactas y mejorar, por tanto, su enseñanza o aprendizaje online (García, Aguaded \& Bartolomé, 2018).

No se puede afirmar que las competencias adquiridas en esos momentos de crisis provocada por la pandemia haya sido la más adecuada por todos los docentes para dar respuesta a la enseñanza a distancia, aunque todo ello se ha sabido revolver de forma inmediata, actuando en el presente con los mejores conocimientos que se poseían, y en un tiempo record. Con un matiz positivo, esto ha posibilitado enfrentar nuevos retos, desafíos e inquietudes para el futuro, pese que la situación vivida de la Covid-19 haya provocado situaciones lamentables e infortunadas a nivel mundial (Según García-Peñalvo et al., 2020)

Con una mirada hacia el devenir, se ha de sacar provecho de esta situación: pasado, presente y futuro; sin olvidar lo comprometido que puede resultar prepararse para algo así, pero teniendo en cuenta que es la sociedad actual la que se está formando para la creación del mañana (López, 2020). Hacer una reflexión de lo que está por llegar y los cambios venideros para la construcción de políticas educativas y actuaciones pedagógicas que se apliquen sin distinción alguna a toda la sociedad, teniendo como premisa el valor de la equidad. También, es necesario recordar el origen de nuestra historia en la enseñanza, para no acometer, así, un yerro en las siguientes generaciones (Arriagada, 2020).

En palabras de Erasmo (citado por Bakker y Wagner, 2020) afirma que "el espacio separa los cuerpos, no las mentes". Esto pone en consideración el actual entramado, vivido y que viviremos. Potenciar la educación a distancia, modalidades de enseñanza como flipped classroom, tutorías entre iguales, trabajo por proyectos, aprendizaje servicio, aprendizaje cooperativo u otras donde se tome en consideración al alumnado como protagonista de su proceso de aprendizaje, podría ser una forma innovadora de enseñar ¿o acaso esto ya existe? De ser así, se tendría que pensar la manera en cómo limitar el espacio-tiempo y el método más adecuado en el que diseñar ésta. De lo contrario, la enseñanza podría revertirse en actuaciones no deseables o en una mala praxis.

En la actualidad, se puede afirmar que los modelos educativos a distancia han supuesto un avance en cuanto a metodología activa y pedagógica y optimización de los recursos tecnológicos se refiere. Sin embargo, aspectos como la evaluación, calificación y obtención de resultados continua siendo un punto débil en este sector, puesto que se dificulta el proceso de verificación, pudiendo infringirse prácticas no deseadas, aunque esto no debería suponer un problema si los docentes considerasen más importantes otros contenidos del currículo oculto, véase la educación en valores, ser éticos, aceptar la diversidad o estar comprometido con lo que cada uno hace (García-Peñalvo et al., 2020).

Para alcanzar fines adecuados a una enseñanza a distancia de calidad, autores cómo DíezGutiérrez \& Gajardo (2020) elaboran pinceladas de lo que comparten las diferentes Comunidades Autónomas, en España, aunque existe controversia respecto a si las actividades deben ser del último trimestre o de anteriores:

"flexibilizar el currículo y las programaciones didácticas", centrando "las actividades lectivas del último trimestre en los aprendizajes y competencias imprescindibles que deberían desarrollar los estudiantes, en función de su etapa, curso, área o materia, renunciando a un cumplimiento 
exhaustivo de los propósitos iniciales, flexibilizando sus planes de trabajo y cuidando de no penalizar o perjudicar el bienestar de su alumnado ni sobrecargarlo de tareas excesivas" (pág.91).

Sin embargo, saber cuáles son las mejores TIC que se ha de emplear en la educación a distancia no define por sí sola las preocupaciones de la enseñanza. Para Morales (2020) existen otros retos educativos a los que hacer frente cuando se habla de tal situación: inteligencia emocional; pensamiento reflexivo; desarrollar enseñanzas centradas en un aprendizaje para la vida; liderazgo y capacidad de superación personal; flexibilidad y adaptabilidad para enfrentar situaciones de cambio. Todo ello sería posible, desde la psicología humanista, acompañando al alumnado en sus aprendizajes, dotándole de la tríada: responsabilidad, autonomía y disciplina que, a su vez, se logra adquirir autorrealización en cada sujeto, al centrarse plenamente en los mismos.

Al igual que en la enseñanza presencial, es necesario resaltar aquellos valores que son fundamentales mantener durante el tiempo, para alcanzar uno de los principales pilares de la educación, cómo es el de saber a convivir. Valores tan importantes y necesarios para tiempos de cambio, donde el caos está presente en nuestros días: tener empatía para aprender a tolerar la situación del prójimo; ayudar para saber darnos a los demás y sentir la alegría que provoca este hecho; cooperar y corresponsabilizar la enseñanza entre escuela y familia para alcanzar sinergias en experiencias y aprendizajes compartidos; ser creativos para resolver una situación problemáticas desde distintos puntos de vista y así, fortalecernos como sociedad; recibir una educación integral, pues la persona se compone de múltiples factores lo que conlleva a adquirir un conocimiento más amplio; razonar de forma dialógica para tolerar el pensamiento diferente, aceptando la diversidad como aspecto común de la sociedad.

En consonancia con lo anterior, poner el valor de lo humano en la educación a distancia y en las TIC se hace necesario para construir una sociedad futura que esté preparada para recibir el futuro próximo y el que aún no estamos preparados a concebir por nuestras limitaciones como humanos. El ingrediente esencial en ello sería la apertura mental de forma global y, en buena medida, en ello puede contribuir las diferentes aplicaciones y software que conviven en el presente. Preparar a la sociedad de hoy para superar las nuevas adversidades, teniendo como potencial el recurso de las TIC.

\section{MÉTODO}

La metodología del presente estudio presenta un corte cuantitativo, donde se ha aplicado un cuestionario ad hoc dirigido tanto a estudiantes como docentes para identificar de manera constructiva las aplicaciones digitales utilizadas durante la época de la Covid-19. Por ende, se ha enviado dicho cuestionario a los centros educativos y universidades de la provincia de Málaga (España). El principal objetivo es analizar el uso que los docentes y estudiantes hacen de las aplicaciones que han utilizado tanto el alumnado como el profesorado en la época Covid-19.

Esta muestra es no probabilística y causal (Alaminos, 2006; Sabariego, 2012), debido a que ha sido de fácil acceso para los investigadores, dado que podía participar cualquier estudiante 0 docente de manera activa con la pretensión de no limitar esta investigación.

Posteriormente, nos pusimos en contacto con el profesorado y los estudiantes por correo electrónico a finales de abril de 2021 para que rellenasen el cuestionario. Tuvimos una breve presentación para explicar el objeto de estudio con la pretensión de que colaboren en esta investigación. Les proporcionamos un enlace para acceder a un cuestionario online, habiendo utilizado la plataforma de Google Forms. Se les garantizó la protección de datos, pues desde el principio se aclaró que las respuestas serían anónimas y tratadas con propósitos de investigación académica de la Universidad de Málaga (España). Para la sistematización de la información se empleó el programa SPSS 24. La medida de adecuación muestral de KMO del cuestionario del alumnado fue de.640 y del profesorado .781, siendo adecuadas para esta investigación. Se analizaron las propiedades psicométricas del instrumento para la validación de este instrumento, teniendo en consideración su fiabilidad y validez. Se aplicó el alfa de Cronbach para el profesorado, obteniéndose un $\alpha=.740$ y en cuanto al alumnado se alcanzó un $\alpha=.658$. Se puede comprobar que presenta una fiabilidad interna aceptable (George y Mallery, 2003). 


\subsection{Objetivos de la investigación}

En el momento en que se declaró el estado de alarma en marzo del año 2020, imposibilitando las clases presenciales, los docentes tuvieron que buscar otras vías para favorecer su formación pedagógica y la inclusión educativa vía online, añadiéndose el manejo de las TIC como un elemento transversal en la enseñanza. En este sentido, los objetivos que se formulan en esta investigación son los siguientes:

- Conocer las aplicaciones que han utilizado el profesorado y el alumnado en época de pandemia.

- Analizar qué aplicaciones se han empleado en las tutorías/seminarios.

- Indagar si han aprendido nuevas aplicaciones digitales durante el estado de alarma.

- Identificar las aplicaciones que suelen aplicar para compartir información.

\subsection{La muestra del estudio}

La muestra del estudio está compuesta por 127 estudiantes de centros educativos y de la Universidad de la provincia de Málaga (España). Ha participado una gran mayoría de 60 alumnos pertenecientes a Grado (47,20\%), seguido de 24 estudiantes de Máster (18,90\%), le prosigue 20 discentes de Bachillerato o Formación Profesional (15,70\%), 17 son de Secundaria $(13,40 \%)$ y solo 6 estudiantes son de Doctorado (4,70\%). Además, han intervenido 86 docentes. Una gran mayoría de 27 docentes (31,40\%) son de Educación Secundaria, 24 profesores (27,90\%) pertenecen a la Universidad, $21(24,40 \%)$ son de Bachillerato o Formación Profesional, 11 $(12,80 \%)$ son de Educación Primaria y una minoría de profesorado, siendo solo $3(3,50 \%)$ son de Educación Infantil.

\subsection{Instrumento}

El cuestionario ad hoc se elaboró una vez que se hizo una revisión bibliográfica de documentos científicos entre los años 2020 y 2021. Se realizaron 10 preguntas, pero se seleccionaron cuatro, debido a que el foco de interés era analizar qué aplicaciones habían utilizado tanto el profesorado como el alumnado para impartir o seguir las clases, tener las tutorías, cuál se tuvo que aprender una vez iniciado el estado de alarma y qué aplicación es la más empleada para compartir información entre el profesorado o el alumnado. En este sentido, el cuestionario está compuesto por preguntas de datos personales como el nivel en el que se encuentran estudiando en caso de ser estudiante o nivel que ejercen para el profesorado. También se plantearon estas preguntas didácticas destinadas tanto al alumnado como al profesorado de la provincia de Málaga (España):

1. ¿Qué aplicación utilizas para seguir tus clases?

2. ¿Qué aplicación utilizas para las tutorías/seminarios?

3. ¿Qué aplicación has tenido que aprender cuando se originó el estado de alarma del $14 / 03 / 2020$ ?

4. ¿Qué aplicación utilizas para compartir información?

\section{RESULTADOS}

Monasterio \& Briceño (2020) indican que el hecho de tener que trasladar las clases presenciales a virtuales supuso un reto tanto al profesorado como al alumnado de los centros educativos y de la Universidad de la provincia de Málaga. Muchos docentes tuvieron muchas dificultades para adaptarse a este inesperado cambio, pues no estaban acostumbrados a programar sus sesiones magistrales de forma no presencial utilizar aplicaciones digitales. En cuanto a los estudiantes, también tuvieron que hacer un esfuerzo para emplear determinadas herramientas digitales, dada a la gran variedad de uso por parte del profesorado Esto es debido a que los docentes buscan aplicaciones donde el alumnado pueda compartir información e interaccionar entre ellos con la pretensión de que las clases sean más dinámicas, activas y capten su motivación. Por ende, ha primado una capacidad de adaptación y flexibilidad para impartir docencia y así el alumnado pudiera seguir con su formación pedagógica en las clases vía online. 
A continuación, se exponen los resultados de los estudios de 125 estudiantes.

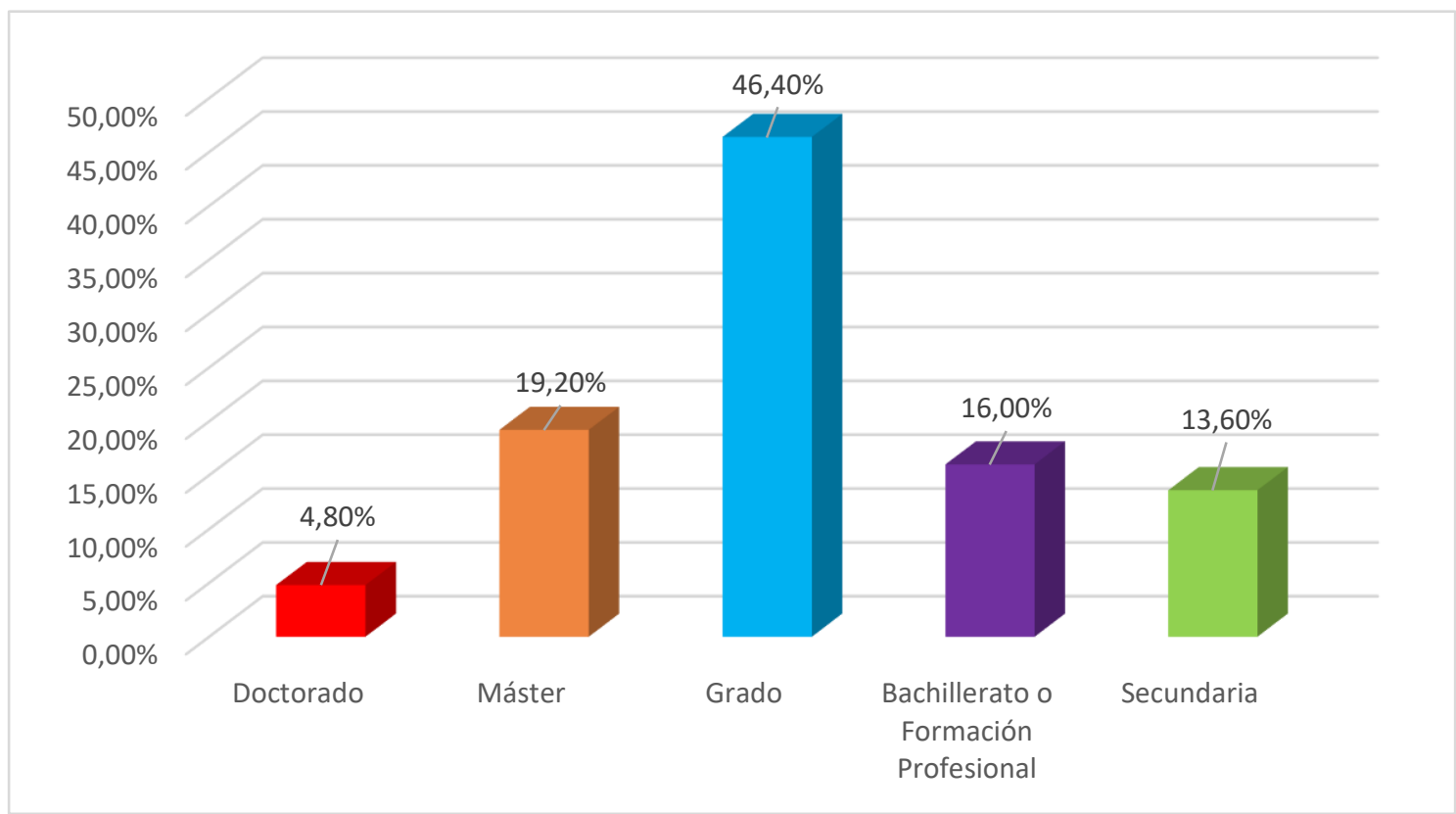

Figura 1. Estudios donde se encuentra el alumnado

Como se puede apreciar en la figura 1, la gran mayoría de estudiantes son de Grado con 58 estudiantes (46,40\%), seguido de 24 estudiantes de Máster (19,20\%), 20 alumnos de Bachillerato o Formación Profesional (16,00\%) y 17 estudiantes de Secundaria (13,60\%). Se deduce que hay un elevado porcentaje de alumnado de Grado.

Estos son los resultados de 86 profesores que se dedican a la docencia.

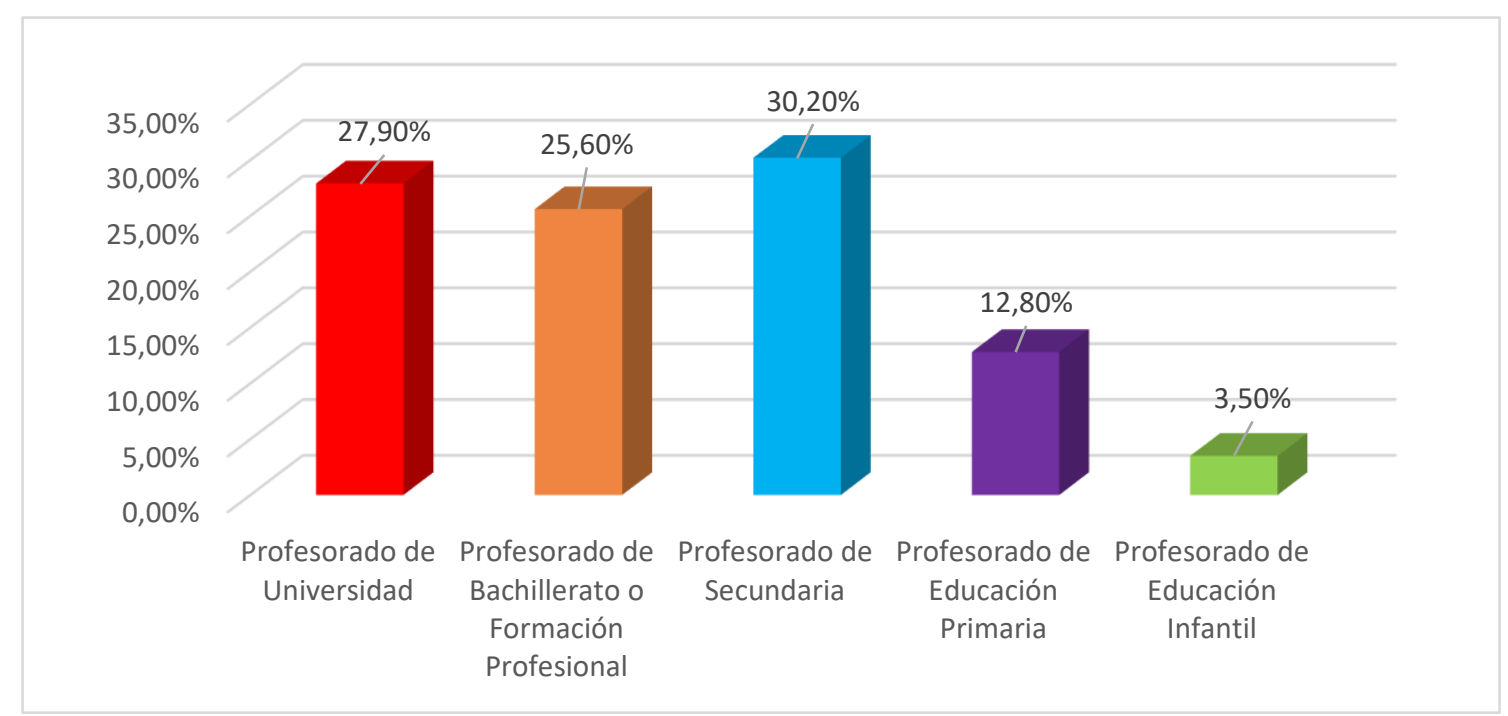

Figura 2. Docencia impartida.

Tal y como se observa en la figura 2, los porcentajes son muy similares entre el Profesorado de Secundaria, siendo 26 (30,20\%), 24 docentes son de Universidad $(27,90 \%), 22$ profesores de Bachillerato o Formación Profesional (25,60\%). Además, han participado 11 docentes de Educación Primaria (12,80\%) y 3 docentes de Educación Infantil (3,50\%).

Una vez que se han apreciado los datos personales del profesorado y del alumnado, se procede a identificar las aplicaciones digitales que utilizan ambos colectivos para seguir las clases, aplicaciones digitales que se utilizan para las tutorías, aplicaciones digitales que se han aprendido durante el estado de alarma del 14/03/2021 y aplicaciones digitales utilizadas para compartir información. 
Tabla 1. Aplicaciones digitales que se utilizan para seguir las clases

\begin{tabular}{|c|c|c|c|c|c|c|}
\hline & \multicolumn{6}{|c|}{ Aplicaciones digitales que se utilizan para seguir las clases ${ }^{1}$} \\
\hline Alumnado & $\begin{array}{c}\text { Google } \\
\text { Meet } \\
(48,00 \%)\end{array}$ & $\begin{array}{c}\text { Moodle } \\
\text { Centros } \\
(15,00 \%)\end{array}$ & $\begin{array}{c}\text { Google Meet, } \\
\text { Blackboard } \\
\text { Collaborate } \\
(7,90 \%)\end{array}$ & $\begin{array}{c}\text { Microsoft } \\
\text { Teams } \\
(5,50 \%)\end{array}$ & $\begin{array}{c}\text { Google } \\
\text { Classroom } \\
(5,50 \%)\end{array}$ & $\begin{array}{c}\text { Zoom } \\
(2,40 \%)\end{array}$ \\
\hline Profesorado & $\begin{array}{c}\text { Moodle } \\
\text { Centros } \\
(17,40 \%)\end{array}$ & $\begin{array}{l}\text { Microsoft } \\
\text { Teams } \\
(14,00 \%)\end{array}$ & $\begin{array}{l}\text { Google } \\
\text { Classroom } \\
(10,50 \%)\end{array}$ & $\begin{array}{c}\text { Google } \\
\text { Classroom, } \\
\text { Google Meet } \\
(5,80 \%)\end{array}$ & $\begin{array}{c}\text { Zoom } \\
(4,70 \%)\end{array}$ & $\begin{array}{c}\text { Webex } \\
(3,50 \%)\end{array}$ \\
\hline
\end{tabular}

Tal y como se aprecia en la tabla 1, tanto el alumnado como el profesorado han aplicado múltiples aplicaciones digitales para seguir las clases en la época de la Covid-19. Un gran porcentaje de estudiantes $(48,00 \%)$ han comentado que suelen utilizar para seguir las clases Google Meet, 19 alumnos (15,00\%) emplean Moodle Centros, 10 estudiantes (7,90\%) prefieren Google Meet, BlackBoard Collaborrate. Son 7 discentes (5,50\%) quienes optan por utilizar Microsoft Teams y otros 7 por Google Classroom. Una minoría de 3 alumnos $(2,40 \%)$ suelen aplicar Zoom. Los docentes también siguen las clases, utilizando aplicaciones digitales similares al alumnado. Son 15 profesores (17,40\%) que aplican Moodle Centros (17,40\%), 12 docentes $(14,00 \%)$ emplean Microsoft Teams, 9 profesores (10,50\%) aplican en sus clases Google Classroom (10,50\%). Un número más reducido como es el caso de 5 docentes $(5,80 \%)$ optan por Google Classroom, Google Meet. Mientras que 3 profesores (4,70\%) eligen Zoom y 3 profesores $(3,50 \%)$ manejan Webex.

Tabla 2. Aplicaciones digitales que se utilizan para las tutorías

\begin{tabular}{|c|c|c|c|c|c|c|}
\hline & \multicolumn{6}{|c|}{ Aplicaciones digitales que se utilizan para las tutorías ${ }^{2}$} \\
\hline Alumnado & $\begin{array}{c}\text { Google } \\
\text { Meet } \\
(45,70 \%)\end{array}$ & $\begin{array}{l}\text { Moodle } \\
\text { Centros } \\
(7,90 \%)\end{array}$ & $\begin{array}{c}\text { Microsoft } \\
\text { Teams } \\
(4,70 \%)\end{array}$ & $\begin{array}{c}\text { Google } \\
\text { Classroom } \\
(4,70 \%)\end{array}$ & $\begin{array}{c}\text { Zoom } \\
(3,90 \%)\end{array}$ & $\begin{array}{c}\text { Blackboard } \\
\text { Collaborate } \\
(3,10 \%)\end{array}$ \\
\hline Profesorado & $\begin{array}{c}\text { Google } \\
\text { Meet } \\
(14,00 \%)\end{array}$ & $\begin{array}{l}\text { Microsoft } \\
\text { Teams } \\
(15,10 \%)\end{array}$ & $\begin{array}{c}\text { Moodle } \\
\text { Centros } \\
(12,80 \%)\end{array}$ & $\begin{array}{c}\text { Google } \\
\text { Classroom } \\
(9,30 \%)\end{array}$ & $\begin{array}{c}\text { Zoom } \\
(5,80 \%)\end{array}$ & $\begin{array}{c}\text { Blackboard } \\
\text { Collaborate } \\
(4,70 \%)\end{array}$ \\
\hline
\end{tabular}

A la luz de los resultados de la tabla 2, se puede comprobar que tanto el alumnado como el profesorado se inclinan por Google Meet, ya que 58 alumnos $(45,70 \%)$ apuestan por esta aplicación y 12 docentes (14,00\%) también emplean esta aplicación con gran frecuencia. En cuanto al alumnado, vemos que hay un descenso notable, debido a que 10 estudiantes $(7,90 \%)$ utilizan la aplicación Moodle Centros. Son 6 alumnos (4,70\%) quienes prefieren emplear Microsoft Teams. Otros 6 alumnos (4,70\%) optan por Google Classroom. Una minoría de 5 alumnos $(3,90 \%)$ aplican el programa Zoom y solo 4 estudiantes tienen interés en utilizan $(3,10 \%)$ Blackboard Collaborate. Se aprecia que 13 docentes (15,10\%) se sirven de Microsoft Teams, 11 profesores $(12,80 \%)$ manejan Moodle Centros. Pocos profesores, como por ejemplo 8 de ellos $(9,30 \%)$ prefieren Google Classroom, 5 docentes $(5,80 \%)$ aplican zoom para el uso de las tutorías y 4 educadores $(4,70 \%)$ prefieren Blackboard Collaborate. Los datos de Google Classroom, Zoom y Blackboard Collaborate son similares al alumnado. Se puede deducir que tanto el alumnado como el profesorado emplean las mismas aplicaciones digitales para el uso de la tutoría.

Tabla 3. Aplicaciones digitales que se han aprendido durante el estado de alarma del 14/03/2020

\begin{tabular}{|c|c|c|c|c|c|c|}
\hline & \multicolumn{6}{|c|}{$\begin{array}{l}\text { Aplicaciones digitales que se han aprendido durante el estado de alarma del } \\
\qquad 14 / 03 / 2021^{3}\end{array}$} \\
\hline Alumnado & $\begin{array}{c}\text { Google } \\
\text { Meet } \\
(22,80 \%)\end{array}$ & $\begin{array}{c}\text { Google } \\
\text { Classroom } \\
(9,40 \%)\end{array}$ & $\begin{array}{l}\text { Zoom } \\
(8,70 \%)\end{array}$ & $\begin{array}{c}\text { Google } \\
\text { Meet, } \\
\text { Blackboard } \\
\text { Collaborate } \\
(7,10 \%)\end{array}$ & $\begin{array}{c}\text { Microsoft } \\
\text { Teams } \\
(4,70 \%)\end{array}$ & $\begin{array}{c}\text { Google } \\
\text { Classroom, } \\
\text { Moodle } \\
\text { Centros } \\
(3,90 \%)\end{array}$ \\
\hline Profesorado & $\begin{array}{c}\text { Microsoft } \\
\text { Teams } \\
(18,60 \%)\end{array}$ & $\begin{array}{c}\text { Google } \\
\text { Classroom } \\
(12,80 \%)\end{array}$ & $\begin{array}{c}\text { Moodle } \\
\text { Centros } \\
(11,60 \%)\end{array}$ & $\begin{array}{l}\text { Zoom } \\
(4,70 \%)\end{array}$ & $\begin{array}{l}\text { Webex } \\
(3,50 \%)\end{array}$ & $\begin{array}{c}\text { Blackboard } \\
\text { Collabore } \\
(2,30 \%)\end{array}$ \\
\hline
\end{tabular}


Como se puede observar en la tabla 3, prácticamente todo el alumnado, siendo 29 estudiantes $(22,80 \%)$ decidieron aprender a utilizar Google Meet durante el estado de alarma del 14/04/2021. Otros estudiantes, es decir, 12 alumnos (9,40\%) profundizaron en Google Classroom $(9,40 \%), 11$ discentes $(8,70 \%)$ se instruyeron en Zoom, 9 educandos $(7,10)$ optaron por aprender Google Meet y Blackboard Collaborate. Un reducido número de alumnado, es decir, $6(4,70 \%)$ prefirieron en aprender Microsoft Teams y 5 alumnos $(3,50 \%)$ apostaron por las aplicaciones de Google Classroom y Moodle Centros. El profesorado ha mostrado especial curiosidad en aprender la aplicación Microsoft Teams, siendo 16 docentes (18,60\%), 11 profesores $(12,80 \%)$ se interesan por Google Classroom, 10 educadores $(11,60 \%)$ por Moodle Centros, 4 docentes $(4,70 \%)$ se inclinan por Zoom, tres profesores $(3,50 \%)$ apuestan por Webex y 2 educadores $(2,30 \%)$ han aprendido en este estado de alarma Blackboard Collaborate. En esta pregunta se puede observar las preferencias de aplicaciones digitales del profesorado y del alumnado.

Tabla 4. Aplicaciones digitales utilizadas para compartir información.

\begin{tabular}{|c|c|c|c|c|c|c|}
\hline & \multicolumn{6}{|c|}{ Aplicaciones digitales utilizadas para compartir información ${ }^{4}$} \\
\hline Alumnado & $\begin{array}{c}\text { Google } \\
\text { Drive } \\
(28,30 \%)\end{array}$ & $\begin{array}{c}\text { Google } \\
\text { Classroom } \\
(9,40 \%)\end{array}$ & $\begin{array}{l}\text { Moodle } \\
\text { Centros } \\
(7,90 \%)\end{array}$ & $\begin{array}{c}\text { Correo } \\
\text { electrónico } \\
(6,30 \%)\end{array}$ & $\begin{array}{c}\text { WhatsApp } \\
(4,70 \%)\end{array}$ & $\begin{array}{l}\text { Twitter } \\
(2,40 \%)\end{array}$ \\
\hline Profesorado & $\begin{array}{c}\text { Google } \\
\text { Classroom } \\
(14,00 \%)\end{array}$ & $\begin{array}{c}\text { Microsoft } \\
\text { Teams } \\
(14,00 \%)\end{array}$ & $\begin{array}{c}\text { Correo } \\
\text { electrónico } \\
(4,70 \%)\end{array}$ & $\begin{array}{c}\text { Moodle } \\
\text { Centros, } \\
\text { Google } \\
\text { Classroom } \\
(3,50 \%)\end{array}$ & $\begin{array}{c}\text { One Drive } \\
(2,30 \%)\end{array}$ & $\begin{array}{c}\text { WhatsApp } \\
\text { Telegram } \\
(1,20 \%)\end{array}$ \\
\hline
\end{tabular}

En lo que respecta a la tabla 4, se puede afirmar que existen numerosas aplicaciones para compartir, 36 estudiantes $(28,30 \%)$ se interesan principalmente por Google Drive, 12 discentes $(9,40 \%)$ prefieren la aplicación Google Classroom, 10 alumnos $(7,90 \%)$ comparten información con Moodle Centros, 8 alumnos (6,30\%) se sirven del correo electrónico, 6 alumnos $(4,70 \%)$ manejan WhatsApp y tres estudiantes $(2,40 \%)$ comparten en la red social de Twitter. El profesorado tiene interés en Google Classroom, en otras palabras 12 docentes $(14,00 \%)$ lo aplican, 12 profesores optan por Microsoft Teams. Al igual que el alumnado, $4(4,70 \%)$ comparten mediante correo electrónico, 3 docentes (3,50\%) se inclinan por Moodle Centros y Google Classroom, 2 educadores (2,30\%) por One Drive y $1(1,20 \%)$ por WhatsApp y Telegram. Las aplicaciones semejantes son Google Classroom, correo electrónico y WhatsApp.

\section{DISCUSIÓN Y CONCLUSIONES}

En esta época de pandemia, el profesorado tuvo la obligación de aprender nuevas vías de aprendizaje, sin haber tenido la oportunidad de una orientación concreta al desarrollo de la actividad docente en la modalidad online. Es posible que muchos docentes hayan participado en acciones formativas en general y concretamente sobre acciones formativas sobre tecnologías en educación.

Además, tuvieron el compromiso ético de ser un guía y un apoyo al alumnado, el cual manifestaba muchas dudas y una gran incertidumbre, dado que nunca se había dado una situación similar en la etapa educativa. Ha habido mucho malestar emocional, aflorando sentimientos de soledad y de impotencia, pues en la era Covid-19, el alumnado apenas puede socializar con sus iguales.

Martínez y Azcona (2020) indican que el profesorado decidió aprender nuevas herramientas y plataformas digitales para una mayor interacción en las aulas digitales, promoviendo la inclusión educativa. En este sentido, el docente ha aplicado múltiples aplicaciones digitales, debido a que pretendía atraer la atención y despertar la curiosidad del alumnado para asimilar los conocimientos pedagógicos de la materia impartida.

En el estado de alarma se han aplicado múltiples aplicaciones digitales, pero se pretende destacar que el alumnado ha empleado principalmente el Google y las redes sociales para seguir las clases en línea, contactar e interaccionar con sus compañeros. Mientras que los docentes han optado por utilizar Microsoft Teams. Esto hace ver que estas aplicaciones y softwares son 
adecuadas para dar una clase no presencial. Sin embargo, cabe reflexionar acerca de la accesibilidad que ofrecen las mismas para todo el alumnado.

En línea con lo anterior, el profesorado está dispuesto a conocer la gamificación para desarrollarla dentro de las aulas, siendo una metodología innovadora, donde predomina el aprendizaje significativo. En este sentido, el alumnado debe estar activo e intervenir en las distintas actividades pedagógicas de gamificación que le proponga el docente, adquiriendo competencias como la digital y aprender a aprender (Trujillo-Torres et al., 2021).

Esta situación hizo que tanto el alumnado como el profesorado se sintieran incómodos, debido a que tuvieron que pasar muchas horas en su casa. Esto ocasionó un malestar emocional como miedo, ansiedad, frustración, impotencia y preocupación sobre lo que sucederá en un futuro no muy lejano. Por ende, el docente debió tener el compromiso de hacer más esfuerzo en la realización de tareas que atrayeran la atención y la motivación del alumnado. Uno de los principales problemas es que el profesor al no tener la presencia física del alumnado no sabe a ciencia exacta si está atento a la lección y trabajando los contenidos demandados (Wang et al., 2020).

Además, estas circunstancias se agravaban con la sobrecarga de tareas que algunos docentes planificaban para sus clases, no teniendo en cuenta que la enseñanza online ha sido un reto en el que se ha tenido que aprender a hacer de forma diaria y casi al momento. No debemos olvidar, que tenían que modificar sus programaciones, debido a que las actividades estaban enfocadas a la presencialidad, teniéndose que adaptar los recursos existentes que beneficien o se ajusten a una época de enseñanza Covid-19, manteniendo los mismos objetivos pedagógicos que se habían implementado al principio del curso académico.

Las aplicaciones digitales que más emplea el alumnado de Universidad para seguir las clases o tener las tutorías son Google Meet, Blackboard Collaborate y Zoom. Sin embargo, los estudiantes de Bachillerato o Formación Profesional prefieren utilizar Moodle Centros y Google Classroom. Todos ellos tuvieron que aprender estas aplicaciones en el estado de alarma del 14/03/2020. Existen múltiples aplicaciones para compartir información y trabajar de manera cooperativa. El alumnado universitario prefiere emplear Google Drive, WhatsApp y Twitter. Los estudiantes de Secundaria y Bachillerato o Formación Profesional eligen Moodle Centros, Google Classroom y correo electrónico.

Con respecto al profesorado de colegios y de institutos optan por las aplicaciones de Moodle Centros, Google Classroom para impartir sus clases vía online y tener tutorías con el alumnado. Mientras que el profesorado de Universidad utiliza Microsoft Teams, Zoom y Webex. Todas las aplicaciones mencionadas anteriormente tuvieron que aprenderlas durante el estado de alarma del 14/03/2020. Los docentes de colegios e institutos aplican las aplicaciones de Google Classroom y Moodle Centros para trabajar conjuntamente y compartir información. El docente universitario emplea aplicaciones como Microsoft Teams y One Drive. Todos los docentes, independientemente de donde imparta docencia utilizan el correo electrónico.

A la luz de las investigaciones, concebir una educación a distancia sin pensar en el aspecto socioafectivo y emocional de la persona es un hito para el conjunto de la sociedad y el bienestar de la misma. Se hace necesario replantear cuáles son las actitudes y medidas no favorecedoras en este entorno y establecer unos límites que sean consensuados por toda la sociedad para evitar perjuicios colaterales, como por ejemplo el cuidado de un menor o el derecho a recibir su educación, al igual que el resto de sus compañeros.

En definitiva, una educación a distancia debe mantener el respeto a los principios democráticos de convivencia como señala la Constitución Española, donde las habilidades sociales, el diálogo y el intercambio de opiniones nutran a los discentes que se desarrollan como ciudadanos del día a día. Esta cuestión tan fundamental no puede caer en el olvido, ya que es elemental para el fomento de la inclusión educativa en la sociedad actual. 


\subsection{Limitaciones del estudio y futuras líneas de investigación}

Las limitaciones que presenta este estudio están relacionadas al instrumento aplicado, pues son pocos los ítems a contestar. Es indispensable que en la futura línea de investigación se formulen más preguntas pedagógicas con respecto a otras dimensiones didácticas como: dificultades manifestadas por el alumnado en época de pandemia, recomendaciones al docente, inconvenientes del profesorado, etc. Además, de hacer entrevistas semiestructuradas tanto al discente como al profesorado para indagar en el estado emocional vivido en la época Covid-19. El tipo de muestreo de conveniencia, es adecuado para el investigador, porque puede participar toda aquella persona que así lo desee, pero tiene sus limitaciones desde una perspectiva metodológica, debido a que generalmente se aplica una muestra aleatoria o probabilística para dar más rigurosidad a la investigación.

La investigación se ha enfocado en los centros educativos y en la Universidad de la provincia de Málaga. La futura línea de investigación sería aplicar este cuestionario a otros centros educativos y a otras universidades para analizar las percepciones y concepciones del profesorado y del alumnado en cuanto a las aplicaciones digitales que se han empleado para fomentar la inclusión educativa dentro de las aulas. Conocer las metodologías de aprendizaje online y las estrategias aplicadas en otras localidades y países contribuye al desarrollo de una educación que se dispone al servicio de toda la comunidad educativa.

\section{ENLACES}

Una vez que se hizo una revisión bibliográfica sobre los aspectos determinantes en la pandemia. Se decidió elaborar un instrumento de los cuestionarios ad hoc sobre aplicaciones digitales en la época de la Covid-19 dirigidos tanto al alumnado como al profesorado, ya que lo consideramos de interés para la comunidad científica.

Enlace al cuestionario para estudiantes: $\underline{\text { https://bit.ly/2UMEeX5 }}$

Enlace al cuestionario para docentes: https://bit.ly/3gy5LD7

\section{REFERENCIAS BIBLIOGRÁFICAS}

Alaminos, A. (2006). El muestreo en la investigación social. En A. Alaminos y J.L. Castejón, Elaboración, análisis e interpretaciónde encuestas, cuestionarios y escalas de opinión, 46-67. Alcoy.

Alonso, C. (8 de julio de 2019). La "implicación parental en la escuela" es una relación de fuerzas entre padres, hijos y profesores. Entramados Sociales. https://bit.ly/3B2iS7n

Anguita, J.M., Méndez, M. y Méndez, D. (2020). Motivación de alumnos de Educación Secundaria y Bachillerato hacia el uso de recursos digitales durante la crisis del Covid-19. Revista Estilos de Aprendizaje, 13, 68-81.

Arriagada, P. (2020). Pandemia Covid-19: Educación a Distancia. O las Distancias en la Educación. Revista Internacional de Educación para la Justicia Social, 9(3), 1-3.

Bakker A. y Wagner D. (2020). Pandemic: lesson for today and tomorrow? Educational Studies in Mathematics, 104, 1-4. https://doi.org/10.1007 / s10649-020-09946-3

Calero, C. (2019). La llegada de las nuevas tecnologías a la educación y sus implicaciones. International Journal of New Education, 2(2). https://doi.org/10.24310/ijne2.2.2019.7449

Díez-Gutiérrez, E.J. y Gajardo, K. (2020). Políticas Educativas en Tiempos de Coronavirus: La Confrontación Ideológica en España. Revista Internacional de Educación para la Justicia Social, 9(3e), 83-101. https://doi.org/10.15366/riejs2020.9.3.005

García-Fernández, N., Rivero, M.L. y Ricis, J. (2020). Brecha digital en tiempo del COVID19. Revista Educativa Hekademos, 28, 76-85. 
García-Peñalvo, F.J., Corell, A., Abella-García, V. y Grande, M. (2020). La evaluación online en la educación superior en tiempos de la COVID-19. Education in the Knowledge Society, 12, https://doi.org/10.14201/eks.23013

García Ruiz, R., Aguaded Gómez, J.I. y Bartolomé Pina, A.R. (2018). La revolución del blended learning en la educación a distancia. Revista Iberoamericana de Educación a Distancia, 21(1), 25-32. https://doi.org/10.5944/ried.21.1.18842

George, D. y Mallery, P. (2003). SPSS for Windows step by step: A simple guide and reference. 11.0 update (4th ed.). Allyn y Bacon.

Gregorio, I. (2020). La Pedagogía Alternativa en Tiempos de Crisis: El Caso de la École Du Dialogue. Revista Internacional de Educación para la Justicia Social, 9(3e), 1-14.

Hussin, A. y Siddappa, N. (2020). The epidemiology and pathogenesis of coronavirus disease (COVID-19) outbreak. Journal of autoimmunity, 1-4. https://doi.org/10.1016/j.jaut.2020.102433

Instituto Nacional de Estadística. (2020, 16 de noviembre). Equipamiento y uso de TIC en los hogares. INE. https://bit.ly/3sFwIPO

Kuric, S, Calderón-Gómez, D. y Sanmartín, A. (2021). Educación y brecha digital en tiempos del COVID-19. Perfiles y problemáticas experimentadas por el alumnado juvenil para continuar sus estudios durante el confinamiento. Revista de Sociología de la Educación-RASE, 14(1), 63-84. https://doi.org/10.7203/RASE.14.1.18265

Ley Orgánica 3/2020, de 29 de diciembre, por la que se modifica la Ley Orgánica 2/2006, de 3 de mayo, de Educación (LOMLOE). Boletín Oficial del Estado, 340, de 30 de diciembre de 2020, 122868-122953. https://bit.ly/3DGDKmh

López, R. (2020). Reflexiones Educativas para el posCovid-19. Recordando el Futuro. Revista Internacional de Educación para la Justicia Social, 9(3e), 127-140. https://doi.org/10.15366/riejs2020.9.3.007

Martín, E. (1 de abril de 2020). El confinamiento aumenta la desigualdad educativa (y no es culpa de los padres). Entramados Sociales .https://bit.ly/3sEC4VP

Martínez, L. y Azcona, A. (2020). Escuela Post-Covid: La oportunidad de repensar la educación en clave inclusiva. Revista Internacional de Educación para la Justicia Social, 9(3), 1-7. https://doi.org/10.15366/riejs2020.9.3.001

Monasterio, D. y Briceño, M. (2020). Educación mediada por las Tecnologías: Un desafío ante la coyuntura del Covid-19. Observador del Conocimiento, 5, 100-108.

Morales, J. (2020). Oportunidad o Crisis Educativa: Reflexiones desde la Psicología para Enfrentar los Procesos de Enseñanza-Aprendizaje en Tiempos de Covid-19. Revista Internacional de Educación para la Justicia Social, 9(3e), 1-9.

Ramiro, J., Vite, H.A. y Feijoo, J.M. (2020). Uso de la tecnología de información y comunicación y las tecnologías de aprendizaje y conocimiento en tiempos de Covid-19 en la Educación Superior. Revista Conrado, 16(77), 338-345.

Ramón, G. (2020). Marcas de la Pandemia: El Derecho a la Educación Afectado. Revista Internacional de Educación para la Justicia Social, 9(3e), 45-59. https://doi.org/10.15366/riejs2020.9.3.003

Real Decreto 463/2020, de 14 de marzo, por el que se declara el estado de alarma para la gestión de la situación de crisis sanitaria ocasionada por el Covid-19. Boletín Oficial del Estado, 67, 1-15. https://bit.ly/3FG9ska

Robinson, Laura; Schulz, Jeremy; Khilnani, Aneka; Ono, Hiroshi; Cotten, Shelia R.; McClain, Noah; Tolentino, Lloyd; Chen, Wenhong; Huang, Gejun; Casilli, Antonio A.; Tubaro, Paola; Dodel, Matías; Quan-Haase, Anabel; Ruiu, Maria Laura; Ragnedda, Massimo; Aikat, Deb y Tolentino, Natalia (2020): "Digital Inequalities in Time of Pandemic: COVID-19 Exposure Risk Profiles and New Forms of Vulnerability". First Monday, 25(7). https://doi.org/https://doi.org/10.5210/fm.v25i7.10845 
Rodicio-García, M.L., Ríos-de-Deus, M.P., Mosquerra-González, M.J. y Penado, M. (2020). La Brecha Digital en Estudiantes Españoles ante la Crisis de la Covid-19. Revista Internacional de Educación para la Justicia Social, 9(3e), 103-125. https://doi.org/10.15366/riejs2020.9.3.006

Sabariego, M. (2012). El proceso de investigación (parte 2). En R. Bisquerra (coord.), Metodología de la investigación educativa (pp. 127-163). La Muralla.

Trujillo-Torres, J.M., Ramos Navas-Parejo, M., Alcalá del Olmo, M.J. y Santos-Villalba, M.J. (2021). La gamificación como herramienta clave en el desarrollo de competencias sostenibles e inclusivas en la Educación Superior. En G. Gómez-García, C. Rodríguez-Jiménez, M.N. Campos-Soto y M. Ramos Navas-Parejo (eds). Nuevos escenarios educativos: hacia el horizonte 2030 (pp. 10-24). Dykinson.

Wang G., Zhang Y., Zhao J., Zhang J. y Jiang, F. (2020). Mitigate the effects of home confinement on children during the Covid-19 outbreak. The Lancet, 395, 945-947. https://doi.org/10.1016/S0140-6736(20)30547-X

\section{INFORMACIÓN SOBRE LAS AUTORAS}

\section{Estela Isequilla Alarcón \\ Universidad de Málaga}

Profesora de la Facultad de Ciencias de la Educación en el Departamento de Didáctica y Organización Escolar de la Universidad de Málaga. Diplomada en Magisterio de Educación Especial, Graduada en Logopedia, Máster Interuniversitario en Cultura de Paz, Conflictos, Educación y Derechos y Doctora en Inclusión Educativa por la Universidad de Málaga. Miembro del Grupo de Investigación de Innovación y Desarrollo Educativo Inclusivo (HUM-1009): https://bit.ly/3hr76v5 Su investigación se interesa especialmente en la relación de las TIC con la Inclusión Educativa.

Más información sobre el trabajo y publicaciones en: https://www.linkedin.com/in/estelaisequilla / ORCID: https://orcid.org/0000-0002-1560-198X

\section{María Martín-Delgado \\ Universidad de Málaga}

María Martín Delgado es Doctoranda del Programa de Doctorado Psicología por la Universidad de Málaga. Graduada en Pedagogía, Máster en Profesorado en Educación Secundaria Obligatoria y Bachillerato, Formación Profesional y Enseñanza de Idiomas, especialidad en Orientación Educativa, Máster en Atención Temprana 'Dr. Miguel de Linares Pezzi' por la Universidad de Málaga. Colaborada del Grupo de Investigación de Leeduca: $\underline{\text { https://bit.ly/3hr76v5 }}$ Su investigación se centra en las dificultades específicas de aprendizaje como la dislexia y discalculia y su desarrollo en las TIC.

Más información sobre el trabajo publicaciones en: https://es.linkedin.com/in/maria-martindelgado / ORCID:orcid.org/0000-0003-3365-4142

Los textos publicados en esta revista están sujetos a una licencia de Reconocimiento 4.0 España de Creative Commons. Puede copiarlos, distribuirlos, comunicarlos públicamente y hacer obras derivadas siempre que reconozca los créditos de las obras (autoría, nombre de la revista, institución editora) de la manera especificada por los autores o por la revista. La licencia completa se puede consultar en:Licencia Creative Commons Atribución-NoComercial-Compartir por igual 4.0 Internacional. 